\title{
Filamin A Mutation May Be Associated With Diffuse Lung Disease Mimicking Bronchopulmonary Dysplasia in Premature Newborns
}

\author{
Amanda Lord MD, Adam J Shapiro MD, Christine Saint-Martin MD, \\ Martine Claveau MSc LLM NNP, Serge Melançon MD, and Pia Wintermark MD
}

\begin{abstract}
Bronchopulmonary dysplasia (BPD) is a common long-term complication in premature newborns requiring ventilatory support and is the most common cause of chronic diffuse lung disease in this population. We present the clinical course of a premature newborn with a complicated neonatal respiratory course that was initially thought to be related to BPD, but it did not respond to the typical therapies for this condition. Due to the findings of periventricular nodular heterotopia, the diagnosis of a filamin A gene mutation was eventually made, which explained the respiratory pathology of this patient. When time of onset and clinical course do not correlate with typical BPD, one should consider alternative diagnoses in premature infants, including neonatal diffuse lung disease. Key words: bronchopulmonary dysplasia; filamin A (FLNA) mutation; heterotopia; diffuse lung disease; newborn. [Respir Care 2014;59(11):e171-e177. () 2014 Daedalus Enterprises]
\end{abstract}

\section{Introduction}

Pediatric diffuse lung disease, including childhood interstitial lung disease, is a heterogeneous group of lung disorders that are characterized by abnormal gas exchange and diffuse pulmonary infiltrates. ${ }^{1,2}$ In older children, the known causes of diffuse lung disease are similar to those in adults and include infections, environmental exposures, and connective tissue or metabolic diseases. ${ }^{1,2}$

However, among newborns and infants, the causes of diffuse lung disease are different. Bronchopulmonary dysplasia (BPD) is the most common cause of chronic diffuse lung disease in premature newborns. ${ }^{3}$ According

Dr Lord is affiliated with the Department of Pediatrics, Dr Shapiro is affiliated with the Division of Pediatric Respirology, Department of Pediatrics, Dr Saint-Martin is affiliated with the Department of Radiology, Dr Wintermark and Ms Claveau are affiliated with the Division of Newborn Medicine, Department of Pediatrics, and Dr Melançon is affiliated with the Division of Genetics, Department of Pediatrics, Montreal Children's Hospital, McGill University, Montreal, Québec, Canada.

The authors have disclosed no conflicts of interest.

Correspondence: Pia Wintermark MD, Division of Newborn Medicine, Montreal Children's Hospital, 2300 Tupper Street, C-920, Montréal, Québec H3H 1P3, Canada. E-mail: pia.wintermark@bluemail.ch.

DOI: $10.4187 /$ respcare. 02847 to the consensus definition of the National Institute of Child Health and Human Development, ${ }^{4,5}$ BPD is considered significant if supplemental oxygen for at least $28 \mathrm{~d}$ of life or postmenstrual 36 weeks gestation is required. The risk of BPD is inversely related to gestational age and birthweight, with $68 \%$ of infants born at $<29$ weeks and weighing $<1,500 \mathrm{~g}$ developing some form of BPD. ${ }^{3}$ Up to now, no genomic loci or pathways have been identified as causing BPD. ${ }^{6}$ A combination of antenatal, perinatal, and postnatal insults to the lung trigger local and systemic inflammatory responses and subsequent secondary lung injury, resulting in chronic lung disease. ${ }^{7}$ The duration and approach to respiratory support are additional risk factors for the development of $\mathrm{BPD}^{8}$ due to immature lung tissue, barotrauma, and volutrauma resulting from mechanical ventilation, oxidative injury, and pro-inflammatory mediators. ${ }^{7}$ Antenatal glucocorticoids, early surfactant therapy, and protective modalities of ventilation have minimized the severity of lung injury in premature newborns. Treatment remains supportive by limiting lung injury during the period of ventilator dependence, optimizing nutrition, and controlling fluid balance.

We present the case of a premature infant with a complicated neonatal respiratory course initially thought to be related to BPD, but for whom the diagnosis of a filamin A gene mutation was eventually made because of the typical brain magnetic resonance imaging findings. 


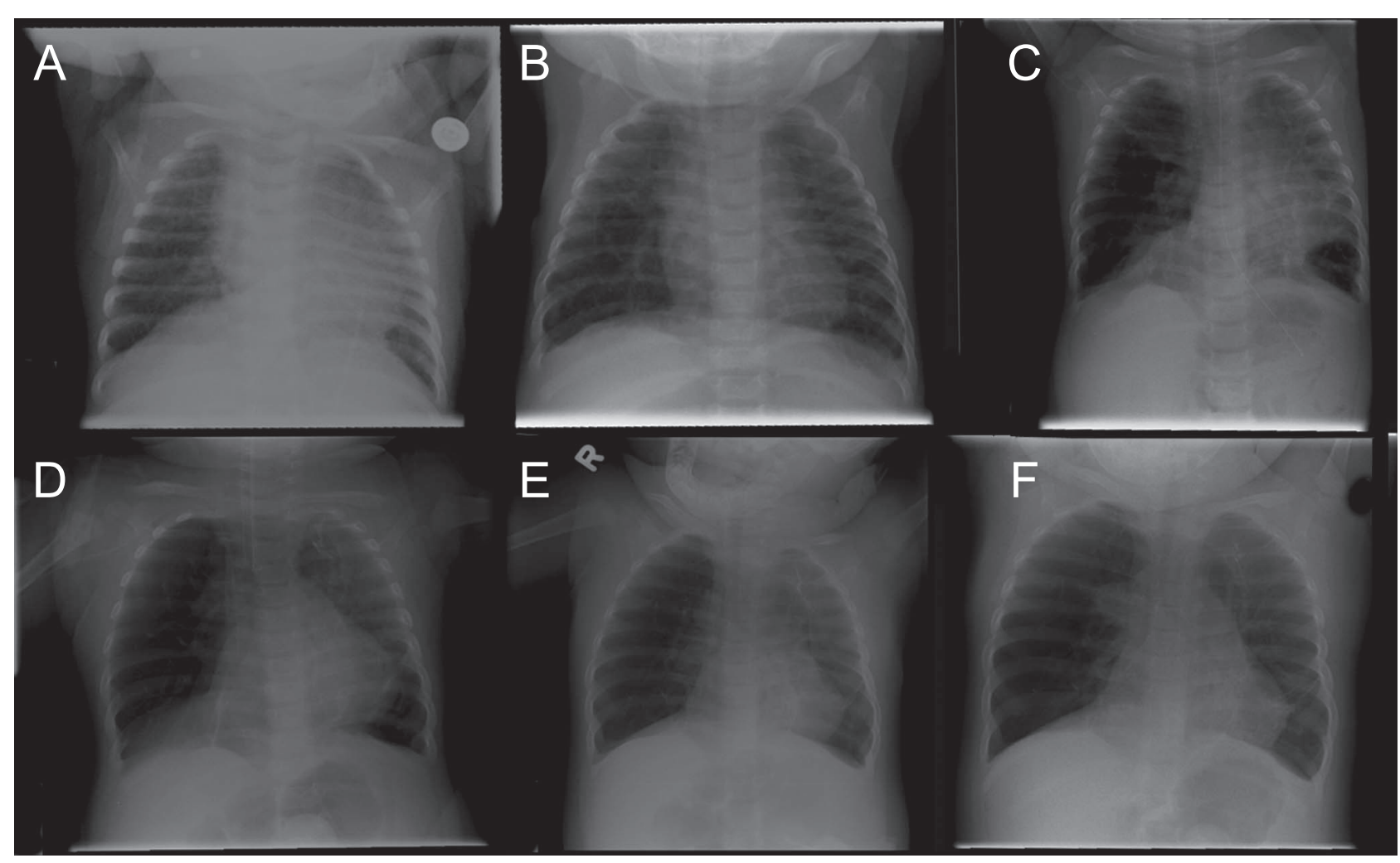

Fig. 1. Serial chest x-rays of our patient. A: day 41 of life (corrected age of 36 weeks). B: day 83 of life (corrected age of 42 weeks). C: 4 months of life (corrected age of 1.5 months). D: 7 months of age (corrected age of 4.5 months). E: 10 months of age (corrected age of 7.5 months). F: 22 months of age (corrected age of 19.5 months). Chest x-ray initially showed only mildly increased diffuse pulmonary infiltrates (A), but evolved into a coarse reticular pattern with moderate hyperinflation (B). Cystic pulmonary lesions progressively developed alternating with heterogeneous areas of atelectasis (C). Areas of atelectasis persisted, as well as right-sided hyperinflation (D-F).

\section{Case Report}

A female infant with a birthweight of $1,730 \mathrm{~g}$ was born via vaginal delivery at 30 weeks of gestation to a 36-y-old mother. The mother had 2 other healthy daughters. The prenatal course was unremarkable, and 2 doses of antenatal betamethasone were given prior to delivery. No clear etiology existed for the premature labor. At delivery, the baby had a good primary adaptation with Apgar scores 7 and 8 at 1 and $5 \mathrm{~min}$, respectively. Initially, the baby was admitted to the neonatal ICU with mild respiratory distress, which completely resolved after $48 \mathrm{~h}$ of supplemental oxygen. No chest x-ray was performed at that time.

Her respiratory status was unremarkable until day 24 of life (corrected age of 33 weeks), when she presented with tachypnea with desaturations requiring supplemental oxygen. Chest X-ray performed on day 41 (Fig. 1A) showed mildly increased diffuse pulmonary infiltrates. She was treated via high-flow nasal cannula at 2-4 $\mathrm{L} / \mathrm{min}$ and with oral diuretics. By day 83 (corrected age of 42 weeks), she had a persistent oxygen requirement and worsening episodes of desaturations with tachypnea and retractions. Chest
X-ray performed on day 83 demonstrated a coarse reticular pattern and moderate hyperinflation (Fig. 1B). Chest computed tomography on day 87 showed a patchy groundglass appearance with areas of hyperinflation and thickening of the interlobular septa (Fig. 2A). A cardiac ultrasound revealed right ventricular hypertrophy and severe pulmonary hypertension with a right atrium-right ventricle gradient of $103 \mathrm{~mm} \mathrm{Hg}$ and a moderate secundum atrial septal defect. She was placed on $100 \%$ oxygen via high-flow nasal cannula, inhaled nitric oxide at $20 \mathrm{ppm}$, sildenafil, and bosentan. In contrast to the degree of oxygen requirement and severe pulmonary hypertension, she had relatively minimal carbon dioxide retention, with a baseline $\mathrm{P}_{\mathrm{CO}_{2}}$ persisting between 40 and $50 \mathrm{~mm} \mathrm{Hg}$.

At 4 months of age (corrected age of 1.5 months), she underwent a cardiac catheterization, confirming severe pulmonary hypertension responsive to oxygen and nitric oxide and showing normal pulmonary vasculature. She progressively developed cystic pulmonary lesions alternating with heterogeneous areas of atelectasis visible on chest x-ray at 4 months (see Fig. 1C). Repeat chest computed tomography showed the persistence of areas of hyperinflation and cystic pulmonary lesions alternating with areas 


\section{Filamin A Mutation Mimicking BPD}

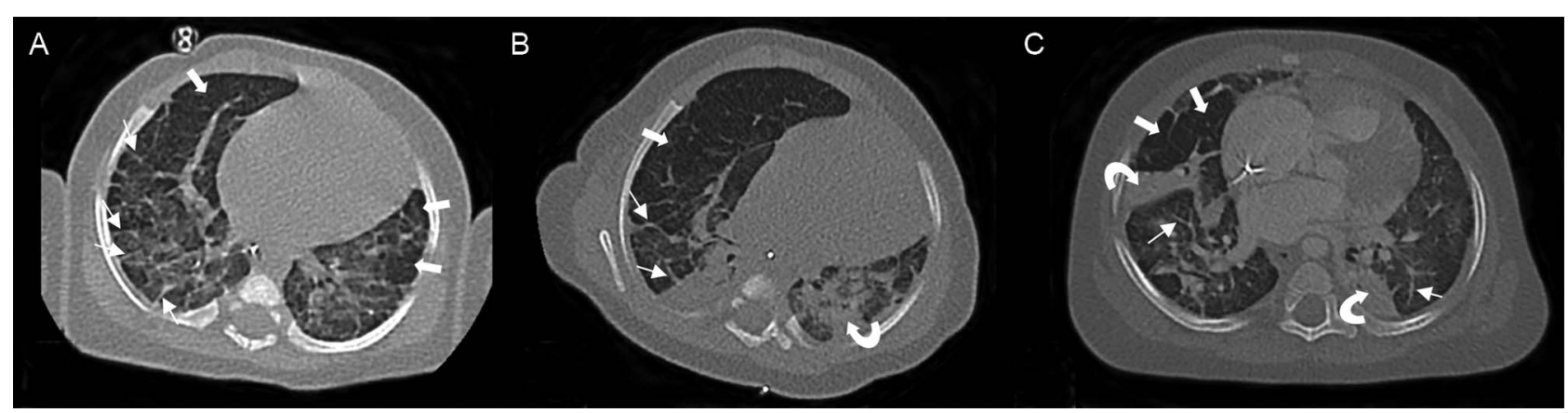

Fig. 2. Serial chest computed tomography of our patient. A: day 87 of life (corrected age of 42 weeks). B: 4 months of age (corrected age of 1.5 months). C: 7 months (corrected age of 4.5 months). Chest computed tomography initially showed a patchy ground-glass appearance with areas of hyperinflation (thick arrows) and thickening of the interlobular septa (thin arrows) (A). The cystic pulmonary lesions alternating with heterogeneous areas of atelectasis (curved arrows) and thickening of the interlobular septa (thin arrows) persisted over time (B and $\mathrm{C})$, but no clearly surgically removable congenital lobar emphysema was apparent.

of atelectasis and thickening of the interlobular septa (see Fig. 2B). Flexible bronchoscopy demonstrated normal lower airway anatomy, and lavage cultures came back positive for cytomegalovirus, which was also isolated in the urine and in nasopharyngeal aspirates at the same time. Bronchoalveolar lavage cultures were otherwise negative. A presumptive diagnosis of cytomegalovirus pneumonitis was made, and she was treated with a 6-week course of ganciclovir. However, her condition did not improve during this treatment. She developed severe coughing episodes with increased respiratory distress, but no other identifiable infectious etiology. Gastroesophageal reflux was suspected, but antacid and promotility agents did not provide improvement.

Due to these increasing episodes of coughing and worsening respiratory distress, the patient was placed on CPAP and then eventually intubated at 5 months of age (corrected age of 2.5 months). Further investigations did not demonstrate any detectable mutations in the main surfactant protein genes (surfactant protein $\mathrm{B}$ or $\mathrm{C}$ or adenosine triphosphate-binding cassette transporter A3). A sweat chloride test was also negative. An open lung biopsy demonstrated mild-to-moderate chronic lung disease, with associated alveolar simplification and pulmonary hypertension, but no evidence of viral inclusions or alveolar capillary dysplasia. Repeat bacterial respiratory cultures were negative. After the open lung biopsy, ventilation became more difficult, and the patient was changed to high-frequency ventilation. A 45-d course of dexamethasone was eventually given, diuretic treatment was optimized, and fluids were restricted, allowing for very slow weaning from ventilatory support. She was extubated to biphasic positive airway pressure at 7 months of age (corrected age of 4.5 months). Her pulmonary arterial hypertension improved to near-normal pressures, and nitric oxide therapy was discontinued, but sildenafil and bosentan were continued.
Repeat chest X-ray showed persistent areas of atelectasis and right-sided hyperinflation, raising the question of congenital lobar emphysema (see Fig. 1D). Repeat chest computed tomography did not confirm surgically correctable congenital lobar emphysema, but instead demonstrated cystic pulmonary lesions alternating with heterogeneous areas of atelectasis (see Fig. 2C). For this reason, along with her slow clinical improvement, no surgical intervention was pursued. She was weaned gradually to CPAP, initially with PEEP of $12 \mathrm{~cm} \mathrm{H}_{2} \mathrm{O}$, and was eventually discharged home at $1 \mathrm{y}$ of age (corrected age of 9.5 months) with CPAP at $8 \mathrm{~cm} \mathrm{H}_{2} \mathrm{O}$, as well as diuretic therapy, sildenafil, and bosentan. Despite residual right-sided hyperinflation on her chest X-ray (see Fig. 1, E and F), she was weaned from CPAP at 19 months of age (corrected age of 16.5 months) and placed on oxygen at $1 \mathrm{~L} / \mathrm{min}$ via nasal prongs. She was completely weaned from oxygen at 22 months of age (corrected age of 19.5 months).

An initial head ultrasound on day 2 of life revealed mild dilatation of the lateral ventricles, which persisted on follow-up ultrasounds. Magnetic resonance imaging of the brain was performed at 7.5 months of age (corrected age of 5 months), which demonstrated bilateral periventricular nodular heterotopia, as well as delayed myelination and widening of subarachnoidal spaces (Fig. 3). Following the findings of periventricular nodular heterotopia, genetic investigations revealed a c.5683G $\rightarrow \mathrm{T}$ transversion in exon 35 of the filamin A gene (FLNA) at chromosomal locus $\mathrm{Xq} 28$. This change, which was also identified in the mother, converts a codon for glutamic acid (GAG) into a termination codon (TAG) and is consistent with a disease-causing mutation. No evidence of a deletion, duplication, or other structural abnormality was detected in the neighboring Xlinked Emery-Dreifuss muscular dystrophy gene. The patient never had clinically evident seizures. At $1 \mathrm{y}$ of age 


\section{Filamin A Mutation Mimicking BPD}

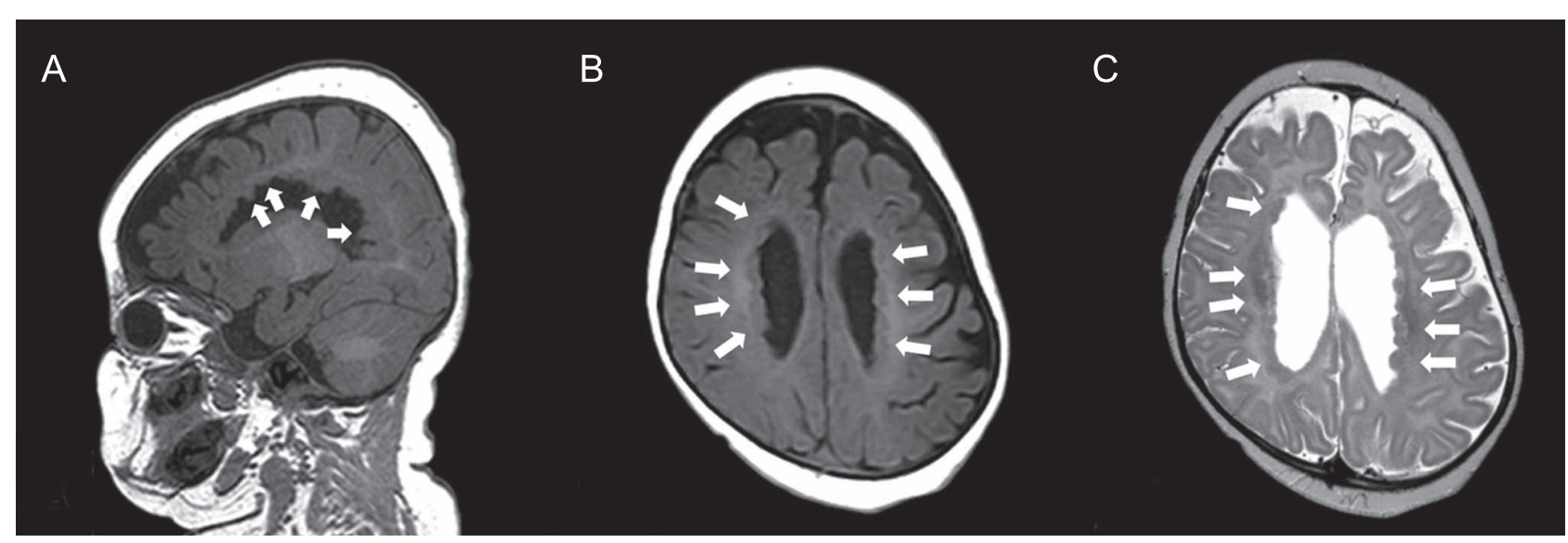

Fig. 3. Cerebral magnetic resonance imaging of our patient performed at $\sim 7.5$ months of age (corrected age of 5 months). A: Sagittal T1-weighted image. B: axial T1-weighted image. C: axial T2-weighted image. Note the presence of bilateral periventricular nodular heterotopia (arrows), as well as widening of subarachnoidal spaces.

(corrected age of 9.5 months), she demonstrated mild axial hypotonia and some degree of gross motor delay.

\section{Discussion}

BPD is the most common cause of chronic diffuse lung disease in premature neonates, but when clinical courses are atypical in timing, severity, and response to standard therapies, other causes of diffuse lung diseases in newborns and infants should be considered and investigated. Our patient presented at birth at 30 weeks of gestation with mild respiratory distress, which completely resolved following $48 \mathrm{~h}$ of supplemental oxygen. Her respiratory status was unremarkable until day 24 of life (corrected age of 33 weeks), when it started to deteriorate and continued to worsen over the next weeks/months, requiring more and more support. Although it cannot be excluded that her prematurity contributed to her more fragile lungs, the presentation was not typical of BPD, which usually develops following respiratory distress after birth and prolonged ventilatory need. Further investigations revealed periventricular nodular heterotopia in the brain. The etiology of periventricular nodular heterotopia is heterogeneous; however, the most common cause of isolated periventricular nodular heterotopia is the $\mathrm{X}$-linked mutation in the filamin A gene (FLNA at chromosomal locus Xq28) that encodes filamin $A,{ }^{9}$ an actin-cross-linking cytoplasmic phosphoprotein that transduces ligand-receptor binding into actin reorganization. This protein is essential for the migration of many cell types, including neuronal, vascular, and cutaneous cells. ${ }^{10}$ Mutations in the filamin A gene are inherited in an X-linked (Xq28) dominant manner, with perinatal lethality in most males. In affected females, intelligence is normal to borderline, and associated neurological features may include seizures of variable severity and onset throughout childhood. ${ }^{11}$ Associated cardiovas- cular anomalies include patent ductus arteriosus, bicuspid aortic valve, and increased risk of stroke due to vasculopathies and large vessel dissection. ${ }^{12}$ Additionally, psychiatric disorders and a hyperlaxity of joints have been reported. ${ }^{13}$

Up to now, only 2 published reports have demonstrated an association between the filamin A gene mutation and pulmonary disease (Table 1). Masurel-Paulet et al ${ }^{14}$ described a 6-y-old boy with a mosaic nonsense filamin A mutation (c.994delG, p.K331X); after being born at term with an uncomplicated perinatal course, he developed dyspnea at 1.5 months of age, which evolved into severe diffuse lung disease. He underwent a subtotal upper lobectomy due to congenital lobar emphysema at 8 months of age, and lung pathology revealed a global absence of bronchial cartilage and hypertensive pulmonary vascular disease. de Wit et al ${ }^{15}$ reported a case of a 3-y-old girl with missense filamin A (c.220G $\rightarrow$ A, p.G74R); she was born at term with an uncomplicated perinatal course, but developed dyspnea at 3 months of age, which evolved into severe diffuse lung disease. She required a lobectomy for right middle lobe emphysema. A third report by GérardBlanluet et $\mathrm{al}^{16}$ described the clinical course of twin boys born at 26 weeks of gestation, a girl born prematurely at 24 weeks of gestation, and another girl born at 35 weeks of gestation to a family with a filamin A mutation $(7922 \mathrm{C} \rightarrow \mathrm{T})$ and periventricular nodular heterotopia. The 3 extremely premature newborns developed respiratory distress from birth and diffuse lung disease thought to be due to BPD and not related to the filamin A mutation since the child born at 35 weeks of gestation did not present any signs of lung disease. Compared with these previous reports, our patient presented an initial benign respiratory course following a preterm birth at 30 weeks, with delayed onset of respiratory symptoms. The clinical course was not consistent with the typical features of BPD of the premature 


\section{Filamin A Mutation Mimicking BPD}

Table 1. Comparison of the Different Cases of Filamin A Mutation Associated With Diffuse Lung Disease in Newborns and Infants

\begin{tabular}{|c|c|c|}
\hline & Masurel-Paulet et al ${ }^{14}$ & de Wit et al ${ }^{15}$ \\
\hline Patients, $n$ & 1 & 1 \\
\hline \multirow[t]{2}{*}{ Genetic mutation } & $\begin{array}{l}\text { Mosaic nonsense filamin A } \\
\text { mutation (c.994delG, } \\
\text { p.K331X) }\end{array}$ & $\begin{array}{l}\text { Missense filamin A mutation } \\
\quad(\text { c. } 220 \mathrm{G} \rightarrow \mathrm{A}, \mathrm{p} . \mathrm{G} 74 \mathrm{R})\end{array}$ \\
\hline & Not present in the mother & Also present in the mother \\
\hline Sex & Male & Female \\
\hline Gestational age & Term & Term \\
\hline Delivery & $\begin{array}{l}\text { Uncomplicated perinatal } \\
\text { course }\end{array}$ & $\begin{array}{l}\text { Uncomplicated perinatal } \\
\text { course }\end{array}$ \\
\hline Initial symptoms & Dyspnea at $1.5 \mathrm{mo}$ of age & Dyspnea at 3 mo of age \\
\hline Evolution & $\begin{array}{l}\text { Severe diffuse lung disease, } \\
\text { including bilateral } \\
\text { pulmonary atelectasis } \\
\text { and cysts, } \\
\text { tracheobronchomalacia, } \\
\text { pulmonary arterial } \\
\text { hypertension, recurrent } \\
\text { respiratory infections, } \\
\text { and prolonged oxygen } \\
\text { dependence }\end{array}$ & $\begin{array}{l}\text { Severe diffuse lung disease, } \\
\text { including bilateral } \\
\text { pulmonary atelectasis and } \\
\text { cysts, bronchomalacia of } \\
\text { the right bronchial tree, } \\
\text { recurrent respiratory } \\
\text { infections, and prolonged } \\
\text { oxygen dependence until } \\
19 \text { mo of age }\end{array}$ \\
\hline X-ray & $\begin{array}{l}\text { Atelectasis alternating with } \\
\text { hyperlucent areas in both } \\
\text { lungs }\end{array}$ & \\
\hline
\end{tabular}

Lung computed Widespread peribronchial tomography thickening, subsegmental collapse, fluid
Severe lobar emphysema of the right middle lobe with compression of the upper lobe

Lobectomy for lobar emphysema at $8 \mathrm{mo}$ of age for lobar emphysema

Lung pathology Global absence of bronchial results cartilage and hypertensive pulmonary vascular disease

Associated features

Follow-up
Periventricular nodular heterotopia with left cerebellar hemisphere hypoplasia and large cisterna magna, patent ductus arteriosus, bifid right urinary drainage system, supraumbilical hernia, and macrothrombocytes
Up to $6 \mathrm{y}$

\section{Periventricular nodular heterotopia with an enlarged retrocerebellar cyst, secundum atrial septal defect, and coarctation of the aorta}

Diffuse lung disease thought to be due to BPD and not related to the filamin A mutation since the child born at $35 \mathrm{wk}$ of gestation did not present any signs of lung disease

Also present in the mother

2 males and 2 females

Premature at $26 \mathrm{wk}$ (male twins), $24 \mathrm{wk}$ (female), and $35 \mathrm{wk}$ (female)

Respiratory distress from birth in the 3 prematurely born newborns, uncomplicated perinatal course for the child born at $35 \mathrm{wk}$ of gestation

Periventricular nodular heterotopia, with microgyria, thin corpus callosum, and reduced white matter in one male twin

\section{Mild respiratory distress completely resolved after $48 \mathrm{~h}$}

Tachypnea with desaturations on day 24 of life (corrected age of $33 \mathrm{wk}$ )

Severe diffuse lung disease, including bilateral pulmonary atelectasis and cysts, tracheobronchomalacia, pulmonary arterial hypertension, and prolonged oxygen dependence until 22 mo of age (corrected age of $19.5 \mathrm{mo}$ )

Areas of hyperinflation alternating with heterogeneous areas of atelectasis

Patchy ground-glass appearance with areas of hyperinflation and cystic pulmonary lesions alternating with areas of atelectasis and thickening of the interlobular septa

Mild-to-moderate chronic lung disease, with associated alveolar simplification and pulmonary hypertension

Periventricular nodular heterotopia
Up to $6 y$, death of one twin at 8 mo of age due to respiratory complications

Up to 22 mo of age (corrected age of $19.5 \mathrm{mo}$ ) 


\section{Filamin A Mutation Mimicking BPD}

Table 2. Causes of Diffuse Lung Disease in Newborns and Infants

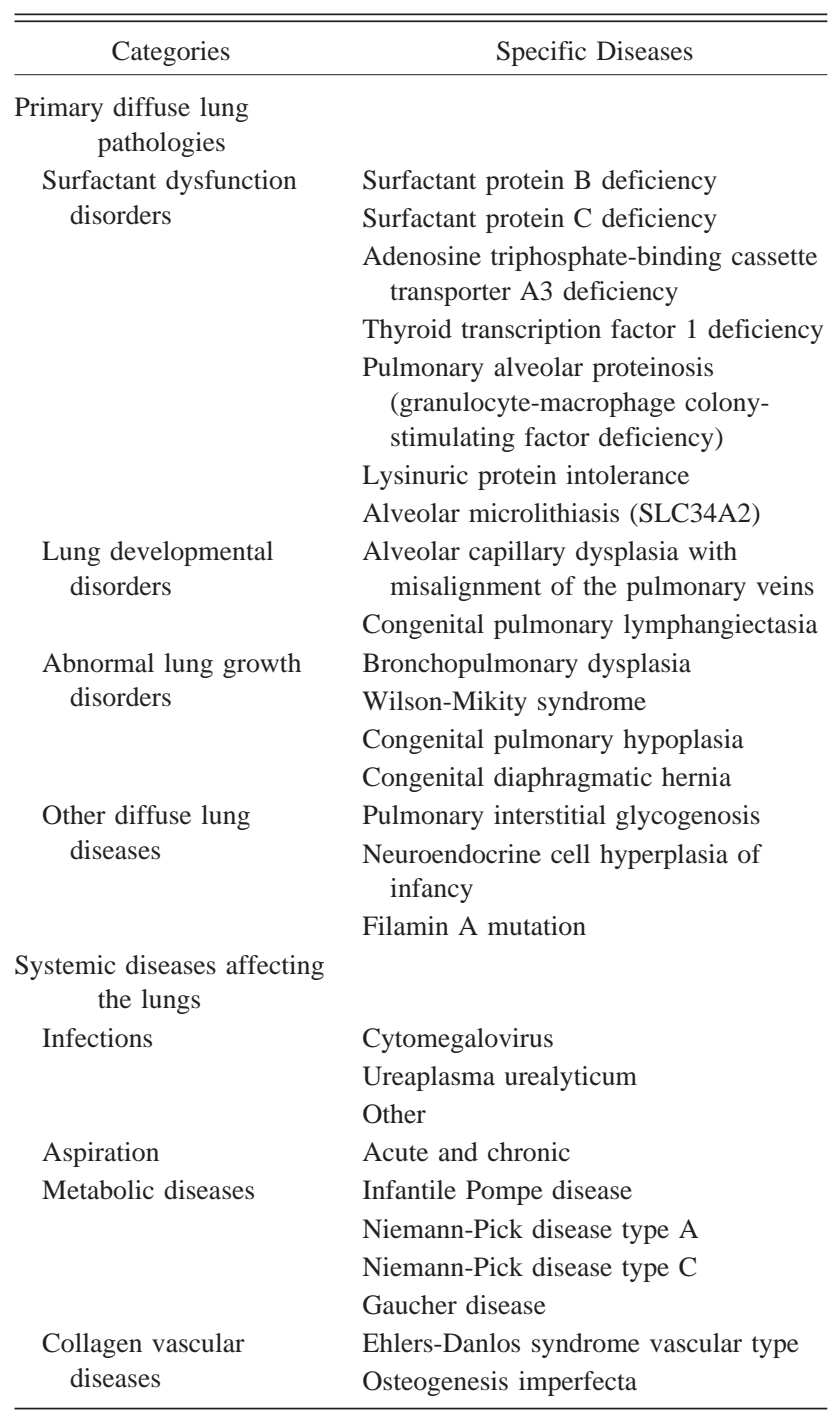

newborn; the delayed onset of respiratory symptoms and the minimal carbon dioxide retention in the context of prolonged oxygen dependence and persistent pulmonary hypertension suggested an alternative etiology. The presence of periventricular nodular heterotopia helped to make the diagnosis in our case.

When faced with diffuse lung disease in an infant that is not clinically typical of standard BPD, caregivers should consider other causes of diffuse lung disease in newborns and infants (Table 2). ${ }^{1,17}$ These disorders include primary lung pathologies and systemic diseases with pulmonary involvement. ${ }^{1,17}$ Among them, the most commonly encountered include surfactant protein deficiency, developmental pulmonary abnormalities, and systemic infections.

Congenitally or perinatally acquired cytomegalovirus pneumonitis is also a rare cause of neonatal respiratory failure that occurs more commonly in premature in- fants. ${ }^{18-20}$ This can result in a complicated ventilatory course and has been associated with the development of BPD, cystic lung disease, congenital lobar emphysema, and severe pulmonary hypertension. A diagnosis is usually made by isolating the cytomegalovirus in the bronchioloalveolar lavage and urine, and a lung biopsy typically demonstrates viral inclusions in pneumocytes. Antiviral agents (eg, ganciclovir) are the recognized treatment modality. ${ }^{18-20}$ In our patient, cytomegalovirus was isolated at some point in the bronchoalveolar lavage. However, despite ganciclovir treatment, her lung condition did not improve, motivating an open lung biopsy that did not show any evidence of viral inclusions. This suggests that cytomegalovirus pneumonitis could have contributed to, but was not the cause of, the diffuse lung disease in our patient. Thus, the mutation in the filamin A gene was the main cause for the diffuse lung disease in our patient.

In conclusion, we presented the case of a premature newborn with a complicated respiratory course, initially thought to be BPD, but for whom the diagnosis of a filamin A mutation was eventually made because of the typical brain magnetic resonance imaging results. Differentiating the typical causes of neonatal respiratory distress and BPD from more rare and complex causes of diffuse lung disease is of the utmost importance because treatment options and prognosis may be quite different in affected newborns and infants.

\section{REFERENCES}

1. Deutsch GH, Young LR, Deterding RR, Fan LL, Dell SD, Bean JA, et al. Diffuse lung disease in young children: application of a novel classification scheme. Am J Respir Crit Care Med 2007;176(11): 1120-1128.

2. Dishop MK. Paediatric lung disease: classification and definitions. Paediatr Respir Rev 2011;12(4):230-237.

3. Stoll BJ, Hansen NI, Bell EF, Shankaran S, Laptook AR, Walsh MC, et al. Neonatal outcomes of extremely preterm infants from the NICHD Neonatal Research Network. Pediatrics 2010;126(3):443-456.

4. Jobe AH, Bancalari E. Bronchopulmonary dysplasia. Am J Respir Crit Care Med 2001;163(7):1723-1729.

5. Ehrenkranz RA, Walsh MC, Vohr BR, Jobe AH, Wright LL, Fanaroff AA, et al. Validation of the National Institutes of Health consensus definition of bronchopulmonary dysplasia. Pediatrics 2005; 116(6):1353-1360.

6. Wang H, St Julien KR, Stevenson DK, Hoffmann TJ, Witte JS, Lazzeroni LC, et al. A genome-wide association study (GWAS) for bronchopulmonary dysplasia. Pediatrics 2013;132(2):290-297.

7. Wilmott RW, Boat TF, Bush A, Chernick V, Deterdin RR, Ratjen F (editors). Kendig and Chernick's disorders of the respiratory tract in children, 8th edition. Philadelphia: Elsevier/Saunders; 2012.

8. Laughon MM, Langer JC, Bose CL, Smith PB, Ambalavanan N, Kennedy KA, et al. Prediction of bronchopulmonary dysplasia by postnatal age in extremely premature infants. Am J Respir Crit Care Med 2011;183(12):1715-1722

9. Clapham KR, Yu TW, Ganesh VS, Barry B, Chan Y, Mei D, et al. FLNA genomic rearrangements cause periventricular nodular heterotopia. Neurology 2012;78(4):269-278. 


\section{Filamin A Mutation Mimicking BPD}

10. Fox JW, Lamperti ED, Ekşioğlu YZ, Hong SE, Feng Y, Graham DA, et al. Mutations in filamin 1 prevent migration of cerebral cortical neurons in human periventricular heterotopia. Neuron 1998;21(6): 1315-1325.

11. Guerrini R, Carrozzo R. Epileptogenic brain malformations: clinical presentation, malformative patterns and indications for genetic testing. Seizure 2001;10(7):532-543.

12. de Wit MC, de Coo IF, Lequin MH, Halley DJ, Roos-Hesselink JW, Mancini GM. Combined cardiological and neurological abnormalities due to filamin A gene mutation. Clin Res Cardiol 2011;100(1):45-50.

13. Sheen VL, Bodell A, Walsh CA. X-linked periventricular heterotopia. In: Pagon RA, Adam MP, Bird TD, Dolan CR, Fong CT, Stephens K, editors. GeneReviews [Internet]. Seattle, WA: University of Washington; 1993-2014. Updated 2009. http://www.ncbi.nlm. nih.gov/books/NBK1213. Accessed June 26, 2014.

14. Masurel-Paulet A, Haan E, Thompson EM, Goizet C, Thauvin-Robinet $\mathrm{C}$, Tai A, et al. Lung disease associated with periventricular nodular heterotopia and an FLNA mutation. Eur J Med Genet 2011; 54(1):25-28.
15. de Wit MC, Tiddens HA, de Coo IF, Mancini GM. Lung disease in FLNA mutation: confirmatory report. Eur J Med Genet 2011;54(3): 299-300.

16. Gérard-Blanluet M, Sheen V, Machinis K, Neal J, Apse K, Danan C, et al. Bilateral periventricular heterotopias in an X-linked dominant transmission in a family with two affected males. Am J Med Genet 2006;140(10):1041-1046.

17. Das S, Langston C, Fan LL. Interstitial lung disease in children. Curr Opin Pediatr 2011;23(3):325-331.

18. Coclite E, Di Natale C, Nigro G. Congenital and perinatal cytomegalovirus lung infection. J Matern Fetal Neonatal Med 2013;26(17): 1671-1675.

19. Sawyer MH, Edwards DK, Spector SA. Cytomegalovirus infection and bronchopulmonary dysplasia in premature infants. Am J Dis Child 1987;141(3):303-305.

20. Walter-Nicolet E, Leblanc M, Leruez-Ville M, Hubert P, Mitanchez D. Congenital cytomegalovirus infection manifesting as neonatal persistent pulmonary hypertension: report of two cases. Pulm Med 2011;2011:293285. 